\title{
The Genus Calophyllum: Review of Ethnomedicinal Uses, Phytochemistry and Pharmacology
}

\author{
Shiv Gupta and Pawan Gupta
}

\begin{abstract}
The species of genus Calophyllum have been reported for several ethnomedicinal uses in the traditional systems of medicine. The scientific study of the genus Calophyllum revealed that it is a rich source of bioactive secondary metabolites. These phytochemicals have shown a wide range of biological activities. Some of these have reached to the clinical developmental stage. The Calophyllum inophyllum seed oil has been proved to be an acceptable sustainable source of biodiesel. Few species of the genus are endangered and have been included in the red list of threatened species by the IUCN Red List. Owing to the importance of the genus a review of its ethnomedicinal uses, phytochemistry, and pharmacology has been carried out. It will further help to explore the molecular mechanism of phytochemicals for health benefits.
\end{abstract}

\section{Keywords}

Coumarins $\cdot$ Xanthones $\cdot$ Antiviral activity $\cdot$ Anti-proliferative activity $\cdot$ HIVinhibitors

\section{$5.1 \quad$ Introduction}

Plant-based natural products have been a potential source of lead compounds for the discovery and development of drugs. Antimalarial drugs such as artemisinin and quinine and anticancer drugs such as paclitaxel and vinblastine are some of the wellknown natural products which are used for the effective treatment of the disease. The major problem associated with the natural products is poor bioavailability and limited yield. These problems are being overcome by medicinal chemists by

S. Gupta $\cdot$ P. Gupta $(\bowtie)$

School of Pharmaceutical Sciences, Lovely Professional University, Phagwara, Punjab, India e-mail: pawan.20270@1pu.co.in 
synthesizing the natural products in good yield and by preparing their analogues with good bioavailability. The genus Calophyllum has also proved to be a potential source of lead compounds for the drug discovery and development. Calanolide A, a non-nucleoside reverse transcriptase inhibitor isolated from the genus, is being evaluated under clinical trial. The genus is a rich source of several medicinally active compounds falling under various chemical classes. The genus includes 190 species and is classified under Calophyllaceae family. The species of the genus are identified with various distinguishing characters like red-coloured outer bark and drupe fruit. The species of the genus such as Calophyllum inophyllum is also known to be used traditionally to alleviate disease and is used in the management of leprosy. Owing to the medicinal importance of the genus Calophyllum, this chapter presents a review of its ethnomedicinal uses, phytochemistry and pharmacology.

\subsection{Morphology and Taxonomy}

The genus Calophyllum was previously included in the Guttiferae family (Group AP 2009). Now, the APG III (Angiosperm Phylogeny Group) system of flowering plant classification classifies it under Calophyllaceae family. There are 190 species in the genus, of which 179 were identified in the Old World (Africa, Asia and Europe) and only 10 species in the New World (the Americas and Oceania) (Eckenwalder 1980). These species are distributed mainly from eastern Africa to the Pacific in the Old World (Stevens 1980). The multivariate analysis by Diaz et al. showed that other American species of the genus Calophyllum are originated from this taxon (Díaz 2013). The species in genus Calophyllum are difficult to classify due to the challenge of establishing distinct boundaries (Watt 2014).

The species under the Calophyllum genus range from very high trees to shrubs. However, most of the species are medium-sized trees. The habitat of the species ranges from wet tropical rainforest of the lowlands to drier areas at higher altitudes. Some of the species are also found in flooded areas. The genus has several distinguishing taxonomical characteristics like red-coloured outer bark with diamond-shaped fissures and presence of opposite leaves with closely and alternating parallel veins. Other characteristics of the species include axillary, terminal and raceme inflorescences. The fruits of the genus Calophyllum are drupe possessing very thin layers of flesh along with a large seed. The sepals and petals in the genus are arranged in hermaphrodite flowers. These also secrete latex which is yellow or white in colour (Eckenwalder 1980). Several species of the genus such as C. apetalum, C. bracteatum, C. caudatum, C. cordato-oblongum and C. mooni have been included in the red list of threatened species by the IUCN RedList. Furthermore, 18 species are categorized as vulnerable (viz. C. apetalum, C. caudatum, C. cordato-oblongum), 5 species as endangered (viz. C. insularum, C. morobense, C. nubicola, C. trapezifolium and C. waliense) and 3 species as critically endangered (viz. C. acutiputamen, C. africanum and C. cuneifolium) (Stevens 1980). 


\subsection{Ethnomedicinal Uses}

A number of plants of the genus Calophyllum are used as traditional medicine for the treatment of chronic diseases such as ulcer, eye infections, haemorrhoids, hypertension, infections, inflammation, leprosy, malaria, nephritis, pain, rheumatism, skin infection, tumours, varicose, venereal diseases, wound and peptic ulcers (Table 5.1). The seed oil of $C$. apetalum is used by traditional practitioners for the treatment of leprosy (Watt 2014). The latex of the seed of $C$. inophyllum has also been used for the management of leprosy. The seed oil of $C$. apetalum and $C$. soulattri was used in the treatment of skin infections (Stevens 1980; Watt 2014). The infusion of C. apetalum mixed with the honey is used for treating scabies (Watt 2014). C. apetalum, C. tacamahaca and C. inophyllum are reported to be used in the treatment of rheumatism (Dorla et al. 2019; Lavergne 2001; Watt 2014). Trunk

Table 5.1 Traditional uses of few species of the genus Calophyllum

\begin{tabular}{|c|c|c|}
\hline Species & Traditional use & References \\
\hline C. apetalum & $\begin{array}{l}\text { Treatment of leprosy and cutaneous infections, infusion } \\
\text { mixed with honey used in scabies and rheumatism }\end{array}$ & Watt (2014) \\
\hline C. blancoi & $\begin{array}{l}\text { Latex used to treat wounds, boils, tumours, swellings and } \\
\text { also to alleviate asthma }\end{array}$ & $\begin{array}{l}\text { Stevens } \\
(1980)\end{array}$ \\
\hline C. brasiliense & $\begin{array}{l}\text { Trunk-bark decoction with the root bark of Coutarea } \\
\text { hexandra used as an antidiabetic and vermifuge, also used } \\
\text { in diarrhoea and intestinal worms }\end{array}$ & $\begin{array}{l}\text { Grenand } \\
\text { et al. (1987), } \\
\text { Yasunaka } \\
\text { et al. (2005) }\end{array}$ \\
\hline C. caledonicum & Diuretic, highly resistant towards fungi and termites & $\begin{array}{l}\text { Hay et al. } \\
(2003) \text {, } \\
\text { Morel et al. } \\
(2002)\end{array}$ \\
\hline C. inophyllum & $\begin{array}{l}\text { Root decoction is used to treat ulcers, boils and } \\
\text { ophthalmia, the bark used to treat orchitis, the latex } \\
\text { rubbed on the skin against rheumatism and psoriasis, and } \\
\text { a leaf decoction to treat eye infections }\end{array}$ & \\
\hline C. lucidum & Dressing of sores, and for a headache remedy & $\begin{array}{l}\text { Abraham } \\
(1912)\end{array}$ \\
\hline C. membranaceum & $\begin{array}{l}\text { Used to reduce inflammation around bruises and to kill } \\
\text { pain, relieve rheumatic joint pain, lumbago and wound } \\
\text { pain }\end{array}$ & \multirow[t]{2}{*}{$\begin{array}{l}\text { Stevens } \\
(1980)\end{array}$} \\
\hline C. soulattri & $\begin{array}{l}\text { Infusion of the root is rubbed on to affected areas in order } \\
\text { to alleviate rheumatic pain, fresh bark from the shoots is } \\
\text { used as medicine for women who have just given birth, oil } \\
\text { obtained from the seed is used externally in the treatment } \\
\text { of rheumatism and skin infections, injected into the } \\
\text { muscles, the refined oil relieves the pain in leprosy }\end{array}$ & \\
\hline C. tacamahaca & $\begin{array}{l}\text { Eye diseases, rheumatism, headache, gout, arthritis, } \\
\text { dermic problems, skin disorders, memory troubles, blood } \\
\text { circulation }\end{array}$ & $\begin{array}{l}\text { Lavergne } \\
\text { (2001) }\end{array}$ \\
\hline C. tomentosum & $\begin{array}{l}\text { Oil extracted from the seed, known as 'kenna tal', is used } \\
\text { in the treatment of skin diseases }\end{array}$ & $\begin{array}{l}\text { Stevens } \\
(1980)\end{array}$ \\
\hline
\end{tabular}


bark decoction of $C$. brasiliense along with the root bark of Coutarea hexandra is used for the treatment of diabetes (Yasunaka et al. 2005). Root decoction of C. inophyllum is used locally in the treatment of ulcers and the leaf decoction is used in the treatment of eye infections (10). Furthermore, infusion of the roots of C. soulattri is rubbed on the skin to alleviate rheumatic pain. The oil extracted from the seeds of $C$. tomentosum is used in the treatment of skin disease (Stevens 1980).

\subsection{Phytochemistry}

The genus Calophyllum is a rich source of bioactive compounds such as xanthones and coumarins. The first phytochemical analysis of the genus was carried out in 1950 by Polonsky and Ormancey-Potier (Ormancey-Potier et al. 1951; Polonsky 1957). Since then several species of the genus have been explored for their phytochemical content. The phytochemical investigation has revealed the presence of various classes of secondary metabolites among which coumarins, xanthones, chromanones, triterpenes, steroid and glycosides are the predominant classes of phytoconstituents present in the genus (Subramanian and Nair 1971; Kashman et al. 1992; McKee et al. 1996; Dharmaratne and Wijesinghe 1997).

\subsubsection{Coumarins}

Coumarins are commonly found in the genus Calophyllum. Most of these coumarins are biosynthesized in the leaves. The coumarins have heterocyclic structure and their biosynthesis is related to the biosynthetic scheme for neo-flavonoids. Coumarins isolated from Calophyllum exhibit various pharmacological activities and can be used as a biomarker. The coumarins of the genus are further subclassified as simple coumarins, furanocoumarins, pyranocoumarins and furo-pyranocoumarins. Calanolide A (1), costatolide (2) (also known as calanolide B), calanolide C (3) and calanolide D (4) were isolated from the fruits and twigs of C. lanigerum. Calanolide E1 (5) and calanolide E2 (6) (diastereoisomer of calanolide E1) were isolated from the stem bark of C. lanigerum (Kashman et al. 1992; McKee et al. 1996). Patil et al. (1993) isolated two tetracyclic dipyranocoumarins, i.e. calophyllic acid (7) and isocalophyllic acid (8), from the leaves of $C$. inophyllum. Similarly, recedesolide (9), a tricyclic pyranocoumarin, was isolated from $C$. blancoi (Shen et al. 2004). Shen et al. (2003) isolated inocalophyllin A (10) and B (12) along with their methyl esters (11and 13, respectively) from the seeds of $C$. inophyllum. Furthermore, Yasunaka et al. (2005), Gomez-Verjan et al. (2014) and Pires et al. (2014) isolated mammea-type coumarins [A/BA (14), A/BB (15) and B/BB (16)] from the leaves of $C$. brasiliense. Tomentolide A (17) and B (18) were isolated from the nut kernels of $C$. tomentosum (Nigam and Mitra 1968). Recently, a new coumarin Wallimarin T (19) was isolated from the stem bark of C. wallichianum (Fig. 5.1; Table 5.2). 
<smiles>CCCc1cc(=O)oc2c3c(c4c(c12)OC(C)(C)C=C4)O[C@H](C)[C@H](C)[C@H]3O</smiles>

Calanolide A 1<smiles>CCCC1CC(=O)Cc2c3c(c(O)c(C(=O)C(C)C(C)O)c21)C=CC(C)(C)O3</smiles>

Calanolide E1<smiles>CCCc1cc(=O)oc2c3c(c4c(c12)OC(C)(C)C=C4)O[C@H](C)[C@H](C)[C@H]3O</smiles>

Calanolide B(Costatolide) 2<smiles></smiles>

Calanolide E2 6<smiles>CCCc1cc(=O)oc2c3c(c4c(c12)OC(C)(C)C=C4)OC(C)[C@H](C)[C@H]3O</smiles>

Calanolide C 3<smiles>CC1C=Cc2c(O)c(/C(=C\C(=O)O)c3ccccc3)c3c(c2C1)C(=O)C(C)CC3(C)C</smiles>

Calophyllic acid 7<smiles>CCCc1cc(=O)oc2c3c(c4c(c12)OC(C)(C)C=C4)O[C@H](C)[C@@H](C)C3=O</smiles>

Calanolide D

4<smiles>CC1C=CC(C)(C)Oc2c(c3c(c(O)c2/C(=C\C(=O)O)c2ccccc2)C(=O)C(C)[C@H](C)C3)C1</smiles>

Isocalophyllic acid<smiles>CCCC1CC(=O)Oc2c(CC=C(C)C)c(O)c3c(c21)O[C@H](C)[C@@H](C)C3=O</smiles>

Recedesolide 9<smiles>C=C(C)C(CC=C(C)C)CC1(CC=C(C)C)C(=O)C(C(CCC)CCC)=C(O)C2=C1OC(C)[C@H](C)C2=O</smiles>

Inocalophyllins B methyl ester<smiles>C=C(C)C(CC=C(C)C)CC1(CC=C(C)C)C(=O)C(C(CC(=O)O)c2ccccc2)=C(O)C2=C1O[C@H](C)[C@H](C)C2=O</smiles>

Inocalophyllin A 10<smiles>CC(C)=CCc1c(O)c(C(=O)CC(C)C)c2oc(=O)cc(-c3ccccc3)c2c1O</smiles>

Mammea A/BA 14<smiles>CCCc1cc(=O)oc2c3c(c4c(c12)OC(C)C(C)C4=O)OC(C)(C)C=C3</smiles>

Tomentolide-B 18<smiles>C=C(C)C(CC=C(C)C)CC1(CC=C(C)C)C(=O)C(C(CC(C)=O)c2ccccc2)=C(O)C2=C1OC(C)C(C)C2=O</smiles>

Inocalophyllins A methyl ester 11<smiles></smiles>

Inocalophyllin B

12<smiles>CC1Oc2c(c3c(c4oc(=O)cc(-c5ccccc5)c24)C=CC(C)(C)O3)C(=O)C1C</smiles>

Tomentolide-A 17<smiles>CCC(C)C(=O)c1c(O)c(CC=C(C)C)c(O)c2c(-c3ccccc3)cc(=O)oc12</smiles>

Mammea A/BB

15<smiles>CCCc1cc(=O)oc2c(C(=O)C(C)CC)c(O)c(CC=C(C)C)c(O)c12</smiles>

Mammea B/BB

16

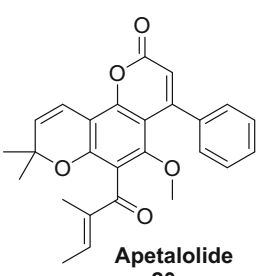

20

Fig. 5.1 Molecular structures (1-132) of bioactive molecules isolated from various species of the genus Calophyllum (1-38: coumarins, 39-79: xanthones, 80-110: chromanones, 111-123: triterpenes and steroids, 124: glycosides, 125-132: miscellaneous compounds) 
<smiles></smiles>

Calophyllolide 21<smiles>CC1(C)C=Cc2c(cc3oc4c(O)cccc4c(=O)c3c2O)O1</smiles>

6-Deoxyjacreubin 1,6-Dihydroxy-5-methoxyxanthone

25<smiles>COc1c(O)ccc2c(=O)c3c(O)cccc3oc12</smiles>
26<smiles>CC1Oc2c(c3c(c4c(-c5ccccc5)cc(=O)oc24)C=CC(C)(C)C(C)O3)C(=O)C1C</smiles>
Inophyllolide 29<smiles>CC1Oc2c3c(c4c(-c5ccccc5)cc(=O)oc4c2OC1(C)C)OC(C)(C)[C@H](C)[C@H]3O</smiles>
Inophyllum H

33<smiles>CC1Oc2c3c(c4c(-c5ccccc5)cc(=O)oc4c2C(=O)C1C)OC(C)(C)C=C3</smiles>

Inophyllum C 30<smiles>CCCC1CC(=O)Oc2c(CC=C(C)C)c(O)c3c(c21)OC(C)C(C)C3=O</smiles>

Isorecedensolide

34<smiles>Cc1cc(=O)oc2c3c(c4c(c12)OC(C)(C)C=C4)OC(C)[C@H](C)[C@H]3O</smiles>

Cordatolide A

23<smiles>Cc1cc(=O)oc2c3c(c4c(c12)OC(C)(C)C=C4)OC(C)[C@H](C)[C@H]3O</smiles>

Cordatolide B

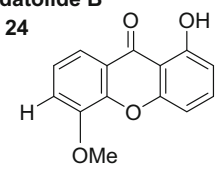<smiles>O=c1c2cc(O)ccc2oc2cccc(O)c12</smiles>

1,7-Dihydroxyxanthone 1-Hydroxy-5-methoxyxanthone 28<smiles>CC1Oc2c3c(c4oc(=O)cc(-c5ccccc5)c4c2OC1C)C=CC(C)(C)C(C)C3O</smiles>

Inophyllum D

31<smiles>C[C@H]1Oc2c3c(c4c(-c5ccccc5)cc(=O)oc4c2C(=O)[C@@H]1C)OC(C)(C)C=C3</smiles>

Inophyllum E

32<smiles>CC1C([Ge])Oc2c3c(c4c(-c5ccccc5)cc(=O)oc4c2C1O)OC(C)(C)C=C3</smiles>

Soulattrolide 37<smiles>COc1cc2oc3c(OC)cccc3c(=O)c2c(O)c1CC=C(C)C</smiles>

1,3,5-Trihydroxy-2-(3methylbut-2-enyl)xanthone 38<smiles>CCCc1cc(=O)oc2c3c(c4c(c12)OC(C)(C)C=C4)OC(C)C(C)C3OC</smiles>

12-Methoxycalanolide A 12-Methoxycalanolide<smiles>CCCc1cc(=O)oc2c3c(c4c(c12)OC(C)(C)C=C4)OC(C)C(C)C3OC</smiles>

36

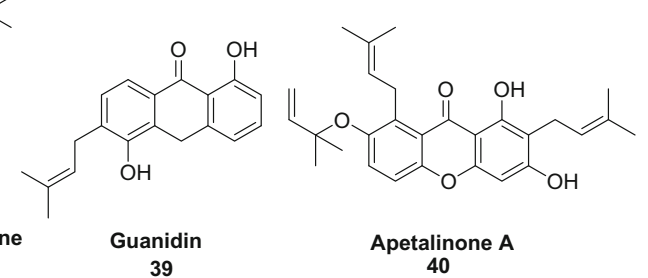

Fig. 5.1 (continued) 
<smiles></smiles>

Acetyl blancoxanthone 41<smiles>COc1ccc2oc3cc4c(c(O)c3c(=O)c2c1CC=C(C)C)C=CC(C)(C)O4</smiles>

Calabaxanthone 42<smiles>C=CC(C)(C)c1c2c(c(O)c3c(=O)c4cccc(O)c4oc13)C=CC(C)(C)O2</smiles>

Caloxanthone C 43<smiles>CC1(C)C=Cc2c(cc3oc4c(O)c(O)ccc4c(=O)c3c2O)O1</smiles>

Jacareubin 44<smiles>CC1(C)C=Cc2c(cc3oc4c(O)cccc4c(=O)c3c2O)O1</smiles>

6-Deoxyjacareubin<smiles>CC(C)=CCc1c2c(c(O)c3c(=O)c4cc5c(c(O)c4oc13)OC(C)(C)C=C5)C=CC(C)(C)O2</smiles>

Caloxanthone

49

Pyranojacareubin

50

51

51<smiles>CC1=CC2c3c(ccc4oc5cc(O)cc(O)c5c(=O)c34)OC(C)(C)C2CC1</smiles>

Calozeyloxanthone

$47 \quad 48$

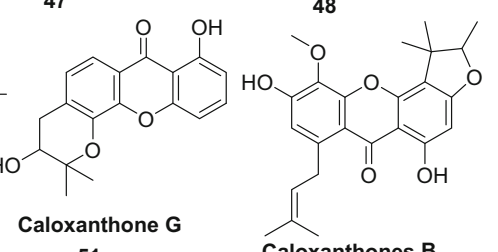

Caloxanthones B<smiles>CC(C)=CCc1c2c(c(O)c3c(=O)c4cc(O)ccc4oc13)C=CC(C)(C)O2</smiles><smiles>COc1cc2oc3c(OC)ccc(O)c3c(=O)c2c(O)c1CC=C(C)C</smiles>

1,3,5-Trihydroxy-2-(3-methylbut - Brasixanthone B 2-enyl)xanthone

56<smiles>CC(C)=CCc1c(O)c(CC=C(C)C)c2oc3ccc4c(c3c(=O)c2c1O)CCC(C)(C)O4</smiles>

Batukinaxanthone

57<smiles>CC(C)=CCc1c2c(c(O)c3c(=O)c4cccc(O)c4oc13)C=CC(C)(C)O2</smiles>

54<smiles>C=C(C)CCC(CC1(CC=C(C)C)C(=O)C2=C(OC(C)C(C)C2=O)C(C(CCC)CC(=O)O)=C1O)C(=C)C</smiles>

Brasiliensic Acid

58<smiles>CC1(C)C=Cc2c(O)c(C(=O)c3ccccc3)c3oc(=O)cc(-c4ccccc4)c3c2O1</smiles>

Calanone

59<smiles>CC(C)=CCc1c2c(c(O)c3c(-c4ccccc4)cc(=O)oc13)C(=O)[C@H](C)[C@@H](C)O2</smiles>

Calaustralin 60

Fig. 5.1 (continued) 


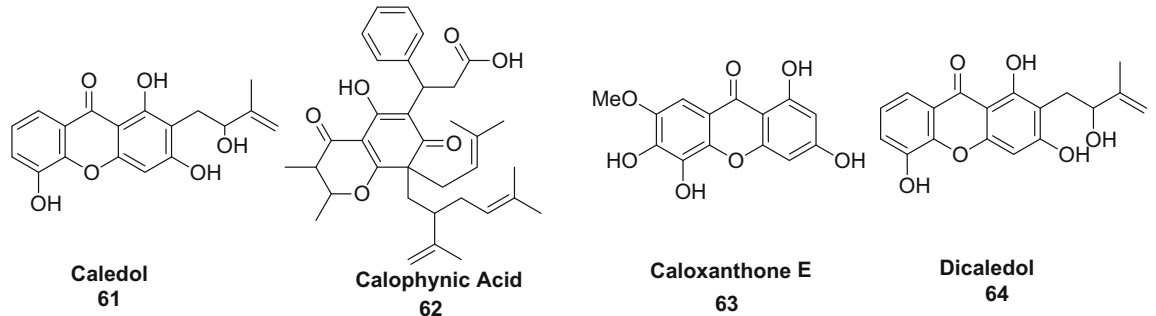<smiles>O=c1c2cccc(O)c2oc2cccc(O)c12</smiles>

1,5-Dihydroxyxanthone 65<smiles>O=c1c2ccccc2oc2c(O)cccc12</smiles>

4-Hydroxyxanthone

69<smiles></smiles>

Rheediaxanthone A 73<smiles>CC(C)=CCc1c(O)cc2oc3c(O)c(O)ccc3c(=O)c2c1O</smiles>

1,3,5,6-Tetrahydroxy-2-(3methyl-2-butenyl)xanthone 76<smiles>CC(C)=CCc1c(O)cc2oc3c(O)c(O)ccc3c(=O)c2c1O</smiles>

1,3,5,6-Tetrahydroxy-2-(3,3-dimethylallyl) xanthone

77<smiles>C=CC(C)(C)c1c2c(c(O)c3c(=O)c4ccc(O)c(O)c4oc13)C=CC(C)(C)O2</smiles>

Macluraxanthone 68
Inophylloidic Acid Phylattrin

71

72<smiles>CC(C)(O)CCc1c(O)cc2oc3c(O)c(O)ccc3c(=O)c2c1O</smiles>

1,3,5,6-Tetrahydroxy-2-(3hydroxy-3-methyl-butyl) xanthone 75<smiles>CC(C)=CCc1c(O)cc2oc3c(O)c(O)ccc3c(=O)c2c1O</smiles>

1,3,5,6-Tetrahydroxy-2isoprenylxanthone 78

Fig. 5.1 (continued) 


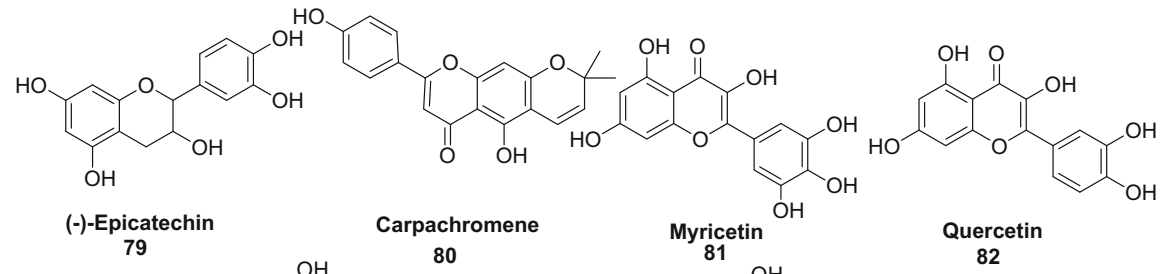

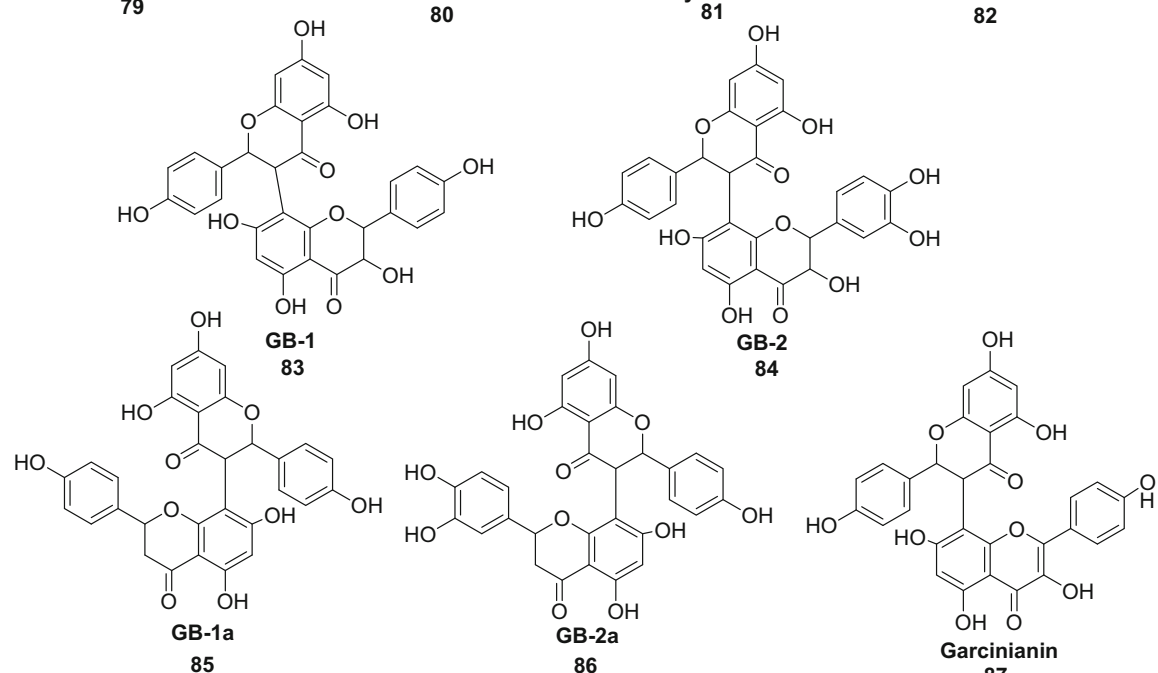

85

86<smiles></smiles>

Pancibiflavonol

88

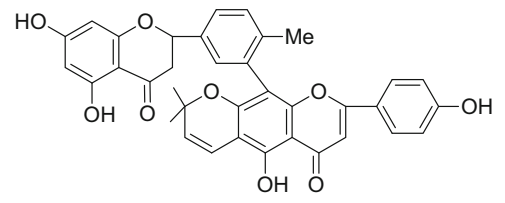

Pyranoamentoflavone 4'-methyl ether 91

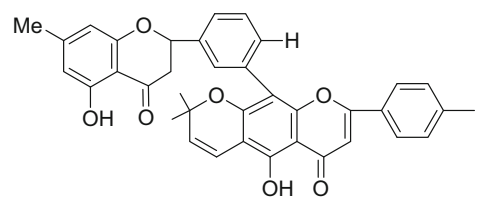

Pyranoamentoflavone 7-methyl ether

92

Fig. 5.1 (continued) 


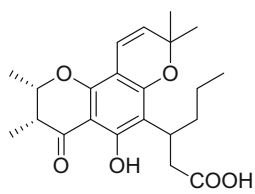

Isocalolongic acid

93<smiles>CC/C=C(/CC(=O)O)c1c2c(c(OC(C)=O)c3c1O[C@H](C)[C@H](C)C3=O)C=CC(C)(C)O2</smiles>

Apetalic acid 5-O-acetate

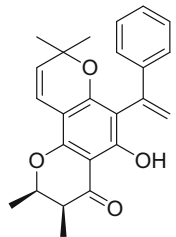

Isoinophynone 94<smiles>CCCC(CC(=O)OC)c1c2c(c(O)c3c1OC(C)C(C)C3=O)C=CC(C)(C)O2</smiles>

Apetalic acid methyl ester<smiles>C=C(c1ccccc1)c1c(O)c2c(c3c1OC(C)(C)C=C3)O[C@H](C)[C@H](C)C2=O</smiles>

Inophynone 95<smiles>CCCC(CC(=O)O)c1c2c(c(O)c3c1OC(C)C(C)C3=O)C=CC(C)(C)O2</smiles>

Apetalic acid 96<smiles>CCCC(CC(=O)O)c1c2c(c(O)c3c1OC(C)C(C)C3=O)C=CC(C)(C)O2</smiles><smiles>CC/C=C(/CC(=O)O)c1c2c(c(OC(C)=O)c3c1O[C@H](C)[C@H](C)C3=O)C=CC(C)(C)O2</smiles>

Isoapetalic acid

Isoapetalic acid 5-0-acetate 98<smiles>[CH2][C@H]1OC2=C(C(CC(=O)O)c3ccccc3)C(=O)C(CC=C(C)C)(C[C@H]3C[C@H](C(=C)C)C3(C)C)C(=O)C2=C(O)[C@H]1C</smiles>

Brasiliensophyllic acid A 103<smiles>CC[C@H]1C[C@H](CC2(CC=C(C)C)C(=O)C3=C(O)[C@@H](C)[C@@H](C)OC3=C(C(CC(=O)O)c3ccccc3)C2=O)C1(C)C</smiles>

Isobrasiliensophyllic acid $\mathrm{A}$<smiles>[CH][C@@H]1O[C@@H]2C(=C(O)[C@@H]1/C=C\I)C(=O)C(CC=C(C)C)(CC(C=C(C)C)CCC(=C)C)C(O)=C2C(CC(=O)O)c1ccccc1</smiles><smiles>C=C(C)[C@H]1C[C@H](CC2(CC=C(C)C)C(=O)C(C(CC(=O)O)c3ccccc3)=C3O[C@H](C)[C@@H](/C=C\C)C(O)C3=C2O)C1(C)C</smiles>

Brasiliensophyllic acid B 105<smiles>CC(C)=CCC1(C[C@@H]2CCC2(C)C)C(=O)C(C(CC(=O)O)c2ccccc2)=C2O[C@H](C)[C@@H](C)C(O)C2=C1O</smiles><smiles>CCCC(C=C(C)C)CC1(CC=C(C)C)C(=O)C2=C(O)[C@@H](C)[C@@H](C)OC2=C(C(CC(=O)O)c2ccccc2)C1=O</smiles><smiles>C/C=C(/c1ccccc1)c1c2c(c3c(c1OC)C(=O)[C@@H](C)[C@H](C)O3)C=CC(C)(C)O2</smiles>

9-hydroxy-2,2,6,7-tetramethyl-2H-[l]benzopyran-(1-phenylethyene-10-yl) -[3,2-b]-dihydropyran-4-one 109
Isobrasiliensophyllic acid B Brasiliensophyllic acid C Isobrasiliensophyllic 106

107<smiles>C=C(C)C1CCC2(C(=O)O)CCC3(C)C(CCC4C5(C)CCC(O)C(C)(C)C5CCC43C)C1CCC1C2(C)CCC2(C)C(C)C(=O)CCC12C</smiles>

\section{Betulinic acid}

110
Canophyllol

111<smiles>CCC12CCC3(C)CCCC3C1CCC1(C)C(C)C(=O)CCC12</smiles>

Friedelin

112

Fig. 5.1 (continued) 


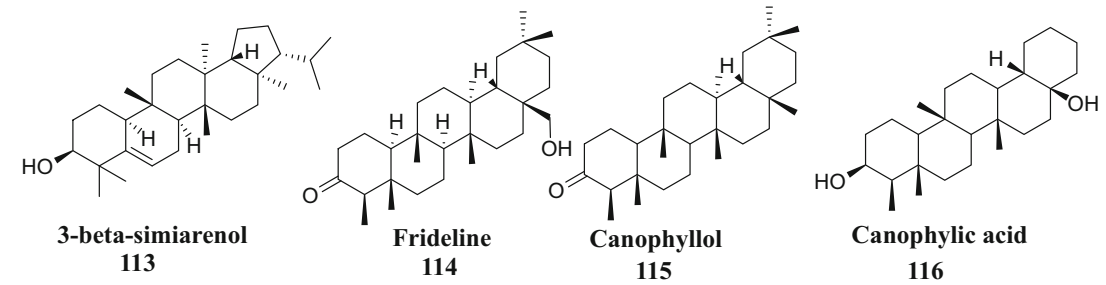

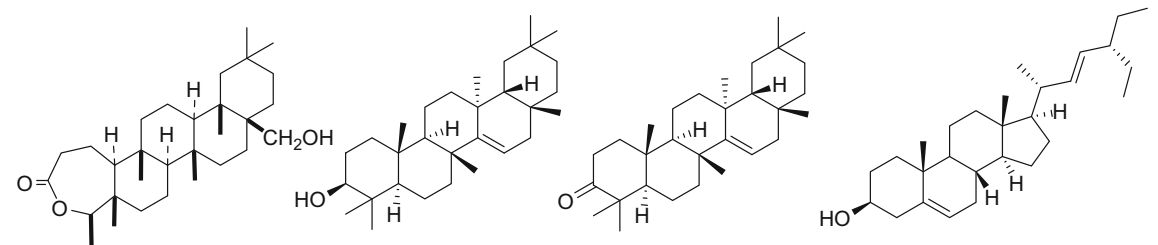

Apetalactone

117

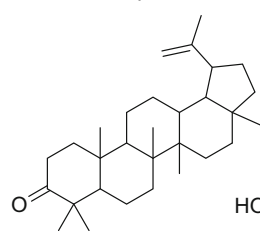

Lupenone

121

\section{Taraxerol}

118

\section{Taraxerone}

119
Stigmasterol

120

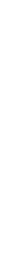<smiles>CCC(C)(O)c1ccc(C(O)OC(O)C(O)CO)c(O)c1</smiles>

Calophymembranside E<smiles>CC(C)=CCC12CC(CCC(C)C)CC3(CCC(C)CCCC(C)C)C(=O)C[C@@H](C1)C(=O)C23C</smiles>

Enervosanone

128<smiles>C/C=C(\C)c1cc(O)c(C2OC(CO)C(O)C(O)C(O)C2O)c(O)c1</smiles>

Calophymembranside C Calophymembranside D 123<smiles>[Z10]c1cc(-c2oc3cc(O[C@H]4C[C@H](CO)[C@@H](O)[C@H](O)[C@H]4O)cc(O)c3c(=O)c2O)cc(O)c1O</smiles>

Calophymembranside $\mathbf{F}$<smiles>C=C(C(=O)c1ccc(O)c(O)c1)C12CC(CC=C(C)C)C(C)(C)C3(CC=C(C)C)C(=O)C(CC=C(C)C)CC13CC(CC=C(C)C)C(C)(C)O2</smiles>

Cambogin

Fig. 5.1 (continued) 

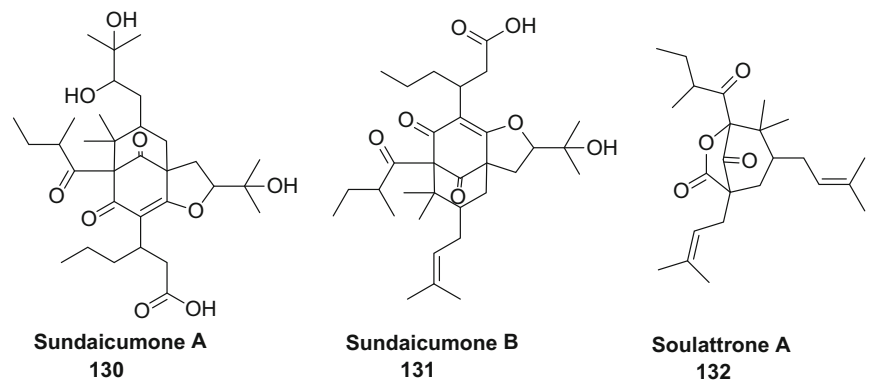

Fig. 5.1 (continued)

\subsubsection{Xanthones}

Xanthones have been isolated from the bark and wood of several species of the genus. These xanthones differ in structure by oxygenation pattern and position of isoprenyl group in the xanthone nucleus. Xanthones are also substituted with various other functionalities like $\mathrm{OH}, \mathrm{OMe}$, OCOMe, 3-carboxybutyl, 1,1-dimethylprop-2enyloxy, 2,3-dihydroxy-3-methylbutyl, 4-hydroxy-3-methylbutyl and 4-hydroxy-3methylbut-2-enyl and 4-hydroxy-3-methylbut-2-enyl. Guanidine (39), a 1,5-dihydroxy-6-(3,3-dimethylbut-2-enyl)-1,5-dihydroxy xanthone, was isolated from the timber of $C$. walker (Dahanayake et al. 1974). Apetalinone A (40) was isolated from $C$. apetalum. Apetalinone A bears 1,1-dimethylprop-2-enyloxy ether substitution which suggested that its biosynthesis involves Claisen rearrangement and Diels-Alder reaction (Iinuma et al. 1997). Acetylblancoxanthone (41), caloxanthone A (42), caloxanthone C (43), jacareubin (44), 6-deoxyjacareubin (45), dombakinaxanthone (46) and osajaxanthone (47) are pyranoxanthones possessing a pyran ring at C5-C6, C6-C7 or C7-C8 isolated from the Calophyllum spp. (Dharmaratne and Wijesinghe 1997; Yimdjo et al. 2004; Shen et al. 2005; Taher et al. 2005; Mah et al. 2015). Dombakinaxanthone (46), a trioxygenated diprenylated chromen-xanthone, and calozeyloxanthone (48) were also isolated from $C$. moonii. (Dharmaratne and Wijesinghe 1997). Furthermore, caloxanthone (49) and pyranojacaeubin (50) possess two pyran rings. Caloxanthone $G(\mathbf{5 1})$ possesses 2,2-dimethyl-3,4-dihydropyrane ring while caloxanthone B (52) possesses a furan ring. Gunasekera et al. (1977) isolated three xanthones calabaxanthone (53), trapezifolixanthone (54) and 1,3,5-trihydroxy-2-(3-methylbut-2-enyl)xanthone (55) from the bark of $C$. cuneifolium. Similarly, brasixanthone B (56) was isolated from the stem bark of $C$. inophyllum (Mah et al. 2015) (Fig. 5.1; Table 5.2).

\subsubsection{Chromanones}

Secondary metabolites having chromanone nucleus, viz. flavonoids, biflavonoids and pyranochromanones, have also been isolated from the genus Calophyllum. 
Table 5.2 Phytochemicals isolated from the species of the genus Calophyllum

\begin{tabular}{|c|c|c|c|}
\hline Compound name & Species & Part used & Reference \\
\hline \multicolumn{4}{|l|}{ Coumarins } \\
\hline Calanolide A & C. lanigerum & $\begin{array}{l}\text { Fruits and } \\
\text { twigs }\end{array}$ & \multirow[t]{4}{*}{$\begin{array}{l}\text { Kashman et al. } \\
(1992)\end{array}$} \\
\hline Calanolide B (Costatolide) & C. lanigerum & $\begin{array}{l}\text { Fruits and } \\
\text { twigs }\end{array}$ & \\
\hline Calanolide $\mathrm{C}$ & C. lanigerum & $\begin{array}{l}\text { Fruits and } \\
\text { twigs }\end{array}$ & \\
\hline Calanolide D & C. lanigerum & $\begin{array}{l}\text { Fruits and } \\
\text { twigs }\end{array}$ & \\
\hline Calanolide E1 & C. lanigerum & Stem bark & \multirow[t]{2}{*}{ McKee et al. (1996) } \\
\hline Calanolide E2 & C. lanigerum & Stem bark & \\
\hline Calophyllic acid & C. inophyllum & Leaves & \multirow[t]{2}{*}{ Patil et al. (1993) } \\
\hline Isocalophyllic acid & C. inophyllum & Leaves & \\
\hline Recedesolide & C. blancoi & Leaves & Shen et al. (2004) \\
\hline Inocalophyllin A & C. inophyllum & Seeds & \multirow[t]{4}{*}{ Shen et al. (2003) } \\
\hline Inocalophyllin A methyl ester & C. inophyllum & Seeds & \\
\hline Inocalophyllin B & C. inophyllum & Seeds & \\
\hline Inocalophyllin B methyl ester & C. inophyllum & Seeds & \\
\hline Mammea A/BA & C. brasiliense & Leaves & $\begin{array}{l}\text { Yasunaka et al. } \\
(2005)\end{array}$ \\
\hline Mammea A/BB & C. brasiliense & Leaves & $\begin{array}{l}\text { Gomez-Verjan et al. } \\
(2014)\end{array}$ \\
\hline Mammea B/BB & C. brasiliense & Leaves & Pires et al. (2014) \\
\hline Tomentolide A & C. tomentosum & Nut kernels & \multirow{2}{*}{$\begin{array}{l}\text { Nigam and Mitra } \\
(1968)\end{array}$} \\
\hline Tomentolide B & C. tomentosum & Nut kernels & \\
\hline Wallimarin $\mathrm{T}$ & C. wallichianum & Stem bark & Tee et al. (2018) \\
\hline Apetalolide & C. apetalum & Nut kernels & $\begin{array}{l}\text { Nigam and Mitra } \\
(1968)\end{array}$ \\
\hline Calophyllolide & C. inophyllum & Kernels & $\begin{array}{l}\text { Ormancey-Potier } \\
\text { et al. (1951) }\end{array}$ \\
\hline 12-Cetoxycalanolide A & C. lanigerum & $\begin{array}{l}\text { Fruits and } \\
\text { twigs }\end{array}$ & Kashman et al. 1992 \\
\hline Cordatolide A & $\begin{array}{l}\text { C. cordato- } \\
\text { oblongum }\end{array}$ & Leaves & \multirow[t]{2}{*}{$\begin{array}{l}\text { Dharmaratne et al. } \\
(1998 a, b)\end{array}$} \\
\hline Cordatolide B & $\begin{array}{l}\text { C. cordato- } \\
\text { oblongum }\end{array}$ & Leaves & \\
\hline 6-Deoxyjacreubin & C. soulattri & Timber & \multirow{4}{*}{$\begin{array}{l}\text { Gunasekera et al. } \\
\text { (1977) }\end{array}$} \\
\hline $\begin{array}{l}\text { 1,6-Dihydroxy-5- } \\
\text { methoxyxanthone }\end{array}$ & C. soulattri & Timber & \\
\hline 1,7-Dihydroxyxanthone & C. soulattri & Timber & \\
\hline 1-Hydroxy-5-methoxyxanthone & C. soulattri & Timber & \\
\hline Inophyllolide & C. inophyllum & Nuts & Kawazu et al. (1968) \\
\hline Inophyllum C & C. inophyllum & Nuts & Yimdjo et al. (2004) \\
\hline Inophyllum D & C. symingtonianum & $\begin{array}{l}\text { Bark and } \\
\text { leaves }\end{array}$ & $\begin{array}{l}\text { Aminudin et al. } \\
(2015)\end{array}$ \\
\hline
\end{tabular}


Table 5.2 (continued)

\begin{tabular}{|c|c|c|c|}
\hline Compound name & Species & Part used & Reference \\
\hline Inophyllum E & C. inophyllum & Nuts & Yimdjo et al. (2004) \\
\hline Inophyllum H & C. symingtonianum & $\begin{array}{l}\text { Bark and } \\
\text { leaves }\end{array}$ & $\begin{array}{l}\text { Aminudin et al. } \\
(2015)\end{array}$ \\
\hline Isorecedensolide & C. blancoi & Roots & Shen et al. (2005) \\
\hline 12-Methoxycalanolide A & C. lanigerum & $\begin{array}{l}\text { Fruits and } \\
\text { twigs }\end{array}$ & \multirow[t]{2}{*}{$\begin{array}{l}\text { Kashman et al. } \\
\text { (1992) }\end{array}$} \\
\hline 12-Methoxycalanolide B & C. lanigerum & $\begin{array}{l}\text { Fruits and } \\
\text { twigs }\end{array}$ & \\
\hline Soulattrolide & C. soulattri & Bark & \multirow{2}{*}{$\begin{array}{l}\text { Gunasekera et al. } \\
\text { (1977) }\end{array}$} \\
\hline $\begin{array}{l}\text { 1,3,5-Trihydroxy-2- } \\
\text { (3-methylbut-2-enyl)xanthone }\end{array}$ & C. soulattri & Timber & \\
\hline
\end{tabular}

\begin{tabular}{|c|c|c|c|}
\hline \multicolumn{4}{|l|}{ Xanthones } \\
\hline Guanidine & C. walker & & $\begin{array}{l}\text { Dahanayake et al. } \\
(1974)\end{array}$ \\
\hline Apetalinone A & C. apetalum & & Iinuma et al. (1997) \\
\hline Acetyl blancoxanthone & C. blancoi & Root & Shen et al. (2005) \\
\hline Caloxanthone A & C. inophyllum & Root bark & Yimdjo et al. (2004) \\
\hline $\begin{array}{l}\text { Caloxanthone C (inoxanthone, } \\
\text { blancoxanthone) }\end{array}$ & C. inophyllum & Stem bark & Mah et al. (2015) \\
\hline Jacareubin & C. brasiliense & Heartwood & \multirow[t]{2}{*}{ Abe et al. (2004) } \\
\hline 6-Deoxyjacareubin & C. brasiliense & Heartwood & \\
\hline Dombakinaxanthone & C. moonii & Root bark & $\begin{array}{l}\text { Dharmaratne and } \\
\text { Wijesinghe (1997) }\end{array}$ \\
\hline Osajaxanthone & C. enervosum & Stem bark & Taher et al. (2005) \\
\hline Calozeyloxanthone & C. moonii & Root bark & $\begin{array}{l}\text { Dharmaratne and } \\
\text { Wijesinghe } 1997\end{array}$ \\
\hline Caloxanthone & C. blancoi & Roots & \multirow[t]{2}{*}{ Shen et al. (2005) } \\
\hline Pyranojacareubin & C. blancoi & Roots & \\
\hline Caloxanthone $\mathrm{G}$ & C. austroindicum & Wood & Iinuma et al. (1996) \\
\hline Caloxanthone B & C. inophyllum & Root bark & Yimdjo et al. (2004) \\
\hline Calabaxanthone & C. cuneifolium & Bark & $\begin{array}{l}\text { Gunasekera et al. } \\
\text { (1977) }\end{array}$ \\
\hline Trapezifolixanthone & C. cuneifolium & Bark & \multirow{2}{*}{$\begin{array}{l}\text { Gunasekera et al. } \\
\text { (1977) }\end{array}$} \\
\hline $\begin{array}{l}\text { 1,3,5-Trihydroxy-2- } \\
\text { (3-methylbut -2-enyl) xanthone }\end{array}$ & C. cuneifolium & Timber & \\
\hline Brasixanthone B & C. inophyllum & Stem bark & Mah et al. (2015) \\
\hline Batukinaxanthone & C. moonii & Root bark & $\begin{array}{l}\text { Dharmaratne and } \\
\text { Wijesinghe (1997) }\end{array}$ \\
\hline Brasiliensic acid & C. inophyllum & Root bark & Yimdjo et al. (2004) \\
\hline Calanone & C. symingtonianum & $\begin{array}{l}\text { Bark \& } \\
\text { leaves }\end{array}$ & $\begin{array}{l}\text { Aminudin et al. } \\
(2015)\end{array}$ \\
\hline Calaustralin & C. inophyllum & Nuts & Yimdjo et al. (2004) \\
\hline Caledol & C. caledonicum & Leaves & Oger et al. (2003) \\
\hline Calophynic acid & C. inophyllum & Root bark & Yimdjo et al. (2004) \\
\hline Caloxanthone $\mathrm{E}$ & C. inophyllum & Root bark & Iinuma et al. (1995) \\
\hline
\end{tabular}


Table 5.2 (continued)

\begin{tabular}{|c|c|c|c|}
\hline Compound name & Species & Part used & Reference \\
\hline Dicaledol & C. caledonicum & Leaves & Oger et al. (2003) \\
\hline 1,5-Dihydroxyxanthone & C. inophyllum & Root bark & \multirow[t]{2}{*}{ Yimdjo et al. (2004) } \\
\hline Friedelan-3-one & C. inophyllum & Root bark & \\
\hline Guanidine (calophyllin B) & C. walkeri & Timber & $\begin{array}{l}\text { Dahanayake et al. } \\
\text { (1974) }\end{array}$ \\
\hline $\begin{array}{l}\text { 3-Hydroxyblancoxanthone } \\
\text { (macluraxanthone) }\end{array}$ & C. blancoi & Roots & Shen et al. (2005) \\
\hline 4-Hydroxyxanthone & C. inophyllum & Stem bark & \multirow[t]{2}{*}{ Mah et al. (2015) } \\
\hline Inophinnin & C. inophyllum & Stem bark & \\
\hline Inophylloidic acid & C. inophyllum & Root bark & Yimdjo et al. (2004) \\
\hline Phylattrin & C. inophyllum & Stem bark & \multirow{3}{*}{ Mah et al. (2015) } \\
\hline Rheediaxanthone A & C. inophyllum & Stem bark & \\
\hline Soulatrin & C. inophyllum & Stem bark & \\
\hline $\begin{array}{l}\text { 1,3,5,6-Tetrahydroxy-2- } \\
\text { (3-hydroxy-3-methyl-butyl) } \\
\text { xanthone }\end{array}$ & C. brasiliense & Heartwood & \multirow[t]{2}{*}{ Abe et al. (2004) } \\
\hline $\begin{array}{l}\text { 1,3,5,6-Tetrahydroxy-2- } \\
\text { (3-methyl-2-butenyl) xanthone }\end{array}$ & C. brasiliense & Heartwood & \\
\hline $\begin{array}{l}\text { 1,3,5,6-Tetrahydroxy-2- } \\
\text { (3,3-dimethylallyl) xanthone }\end{array}$ & C. brasiliense & Heartwood & $\begin{array}{l}\text { Yasunaka et al. } \\
\text { (2005) }\end{array}$ \\
\hline $\begin{array}{l}\text { 1,3,5,6-Tetrahydroxy-2- } \\
\text { isoprenylxanthone }\end{array}$ & C. austroindicum & Trunk & Iinuma et al. (1996) \\
\hline
\end{tabular}

\section{Flavonoid}

\begin{tabular}{|c|c|c|c|}
\hline (-)-Epicatechin & C. enervosum & Stem bark & Taher et al. (2005) \\
\hline Carpachromene & C. symingtonianum & $\begin{array}{l}\text { Bark \& } \\
\text { leaves }\end{array}$ & $\begin{array}{l}\text { Aminudin et al. } \\
(2015)\end{array}$ \\
\hline Myricetin & C. inophyllum & Androecium & \multirow{2}{*}{$\begin{array}{l}\text { Subramanian and } \\
\text { Nair (1971) }\end{array}$} \\
\hline Quercetin & C. inophyllum & Androecium & \\
\hline GB-1 & C. pauciflorum & Stem bark & \multirow[t]{7}{*}{ Ito et al. (1999) } \\
\hline GB-2 & C. pauciflorum & Stem bark & \\
\hline GB-1a & C. pauciflorum & Stem bark & \\
\hline GB-2a & C. pauciflorum & Stem bark & \\
\hline Garcinianin & C. pauciflorum & Stem bark & \\
\hline Pancibiflavonol & C. pauciflorum & Stem bark & \\
\hline GD-IV & C. pauciflorum & Stem bark & \\
\hline Amentoflavone & C. brasiliense & Leaves & $\begin{array}{l}\text { Reyes-Chilpa et al. } \\
\text { (2004) }\end{array}$ \\
\hline $\begin{array}{l}\text { Pyranoamentoflavone } 4^{\prime} \text {-methyl } \\
\text { ether }\end{array}$ & C. venulosum & Leaves & \multirow[t]{2}{*}{ Cao et al. (2001) } \\
\hline $\begin{array}{l}\text { Pyranoamentoflavone 7-methyl } \\
\text { ether }\end{array}$ & C. venulosum & Leaves & \\
\hline \multicolumn{4}{|l|}{ Pyranochromanone } \\
\hline Isocalolongic acid & C. recedens & Bark & $\begin{array}{l}\text { Guerreiro et al. } \\
\text { (1973) }\end{array}$ \\
\hline Isoinophynone & C. inophyllum & Leaves & Khan et al. (1996) \\
\hline
\end{tabular}


Table 5.2 (continued)

\begin{tabular}{|c|c|c|c|}
\hline Compound name & Species & Part used & Reference \\
\hline Inophynone & C. inophyllum & Leaves & Ali et al. (1999) \\
\hline Apetalic acid & C. blancoi & Seeds & \multirow[t]{6}{*}{ Shen et al. (2004) } \\
\hline Apetalic acid 5-O-acetate & C. blancoi & Seeds & \\
\hline Apetalic acid methyl ester & C. blancoi & Seeds & \\
\hline Isoapetalic acid & C. blancoi & Seeds & \\
\hline Isoapetalic acid 5-O-acetate & C. blancoi & Seeds & \\
\hline Isoapetalic methyl ester & C. blancoi & Seeds & \\
\hline Isocordato-oblongic acid & C. symingtonianum & $\begin{array}{l}\text { Bark \& } \\
\text { leaves }\end{array}$ & $\begin{array}{l}\text { Aminudin et al. } \\
\text { (2015) }\end{array}$ \\
\hline Brasiliensophyllic acid A & C. brasiliense & & \multirow{6}{*}{$\begin{array}{l}\text { Cottiglia et al. } \\
(2004)\end{array}$} \\
\hline Isobrasiliensophyllic acid A & C. brasiliense & & \\
\hline Brasiliensophyllic acid B & C. brasiliense & & \\
\hline Isobrasiliensophyllic acid B & C. brasiliense & & \\
\hline Brasiliensophyllic acid C & C. brasiliense & & \\
\hline Isobrasiliensophyllic acid A & C. brasiliense & & \\
\hline $\begin{array}{l}\text { 9-Hydroxy-2,2,6,7-tetramethyl- } \\
\text { 2H-[1]-benzopyran- } \\
\text { (1-phenylethyene-10-yl)-[3,2-b]- } \\
\text { dihydropyran-4-one }\end{array}$ & C. tomentosum & Leaves & Babu et al. (1994) \\
\hline
\end{tabular}

\section{Triterpenes and steroids}

\begin{tabular}{|c|c|c|c|}
\hline Betulinic acid & C. tomentosum & Bark & $\begin{array}{l}\text { Karunanayake et al } \\
\text { (1981) }\end{array}$ \\
\hline Canophyllol & C. brasiliense & Leaves & $\begin{array}{l}\text { Reyes-Chilpa et al. } \\
(2004)\end{array}$ \\
\hline Friedelin & C. brasiliense & Leaves & $\begin{array}{l}\text { Yasunaka et al. } \\
\text { (2005) }\end{array}$ \\
\hline $3 \beta$-Simiarenol & C. walkeri & & $\begin{array}{l}\text { Dahanayake et al. } \\
\text { (1974) }\end{array}$ \\
\hline Frideline & C. apetalum & & \multirow[t]{2}{*}{ Joshi et al. (2013) } \\
\hline Canophyllol & C. apetalum & & \\
\hline Canophyllic acid & C. inophyllum & & \multirow{2}{*}{$\begin{array}{l}\text { Govindachari et al. } \\
\text { (1968), } \\
\text { Govindachari et al. } \\
\text { (1967) }\end{array}$} \\
\hline Apetalactone & C. apetalum & & \\
\hline Taraxerol & C. walkeri & & $\begin{array}{l}\text { Dahanayake et al. } \\
\text { (1974) }\end{array}$ \\
\hline Taraxerone & C. moonii & & $\begin{array}{l}\text { Dharmaratne and } \\
\text { Wijesinghe (1997) }\end{array}$ \\
\hline Stigmasterol & C. wallichianum & Stem bark & Tee et al. (2018) \\
\hline Lupenone & C. symingtonianum & $\begin{array}{l}\text { Bark \& } \\
\text { leaves }\end{array}$ & $\begin{array}{l}\text { Aminudin et al. } \\
\text { (2015) }\end{array}$ \\
\hline Sitosterol & C. apetalum & Bark & $\begin{array}{l}\text { Nigam and Mitra } \\
\text { (1969) }\end{array}$ \\
\hline \multicolumn{4}{|l|}{ Glycoside } \\
\hline Calophymembranside $\mathrm{C}$ & C. membranaceum & Stem & Ming et al. (2016) \\
\hline
\end{tabular}


Table 5.2 (continued)

\begin{tabular}{|c|c|c|c|}
\hline Compound name & Species & Part used & Reference \\
\hline Calophymembranside D & C. membranaceum & Stem & \multirow[t]{3}{*}{ Zhu et al. (2018) } \\
\hline Calophymembranside E & C. membranaceum & Stem & \\
\hline Calophymembranside F & C. membranaceum & Stem & \\
\hline Myricetin-7-glucoside & C. inophyllum & Androecium & $\begin{array}{l}\text { Subramanian and } \\
\text { Nair (1971) }\end{array}$ \\
\hline \multicolumn{4}{|l|}{ Miscellaneous compounds } \\
\hline Enervosanone & C. enervosum & Stem bark & \multirow[t]{2}{*}{ Taher et al. (2005) } \\
\hline Cambogin & C. enervosum & Stem bark & \\
\hline Sundaicumone A & C. sundaicum & Leaves & \multirow[t]{2}{*}{ Cao et al. (2006a, b) } \\
\hline Sundaicumone B & C. sundaicum & Leaves & \\
\hline Soulattrone A & C. soulattri & Bark & Nigam et al. (1988) \\
\hline
\end{tabular}

Epicatechin $(\mathbf{8 0})$, carpachromene $(\mathbf{8 1})$ and myricetin $(\mathbf{8 2})$ flavonoids have been isolated from the stem bark, leaves and androecium of flowers, from C. enervosum, C. symingtonianum and C. inophyllum, respectively (Table 5.2) (Subramanian and Nair 1971; Taher et al. 2005; Aminudin et al. 2015). Quercetin (83) was also isolated from the androecium of flowers of C. inophyllum (Subramanian and Nair 1971).

The biflavonoids of type flavanone-flavonol [GB-1 (84) and GB-2 (85)], flavanoneflavanone [GB-1a (86) and GB-2a (87)], flavanone-flavonol [garcinianin (88) and pancibiflavonol (89)], flavanone-flavone [GD-IV (90)] and flavone-flavone [amentoflavone (91)] have also been isolated from the species of genus Calophyllum (Table 5.2) (Ito et al. 1999; Reyes-Chilpa et al. 2004). Pyranoamentoflavone 4'-methyl ether (92) and pyranoamentoflavone 7-methyl ether $(\mathbf{9 3})$ were also isolated from the leaves of $C$. venulosum (Cao et al. 2001).

Isocalolongic acid (94), isoinophynone (95) and inophynone (96) are 1-benzopyran-4-one class of compounds possessing an additional pyran ring which is fused at C7-C8 bond. Inophynone (96) and isoinophynone (95) which are a pair of epimers were isolated from the ethanolic extract of the fresh leaves of C. inophyllum (Ali et al. 1999). Compounds 97-103 are pyranochromanone derivatives, isolated from various species of the genus Calophyllum (Table 5.2) (Shen et al. 2004; Aminudin et al. 2015).

Brasiliensophyllic acid A (104), isobrasiliensophyllic acid A (105), brasiliensophyllic acid B (106), isobrasiliensophyllic acid B (107), brasiliensophyllic acid C (108) and isobrasiliensophyllic acid A (109) are novel chromanone acids which were isolated from the bark of $C$. brasiliense (Cottiglia et al. 2004) (Fig. 5.1; Table 5.2).

\subsubsection{Triterpenes and Steroid}

Betulinic acid (111), canophyllol (112) and friedelin (113) are the most common triterpenes found in the genus Calophyllum (Table 5.2) (Karunanayake et al. 1981; 
Reyes-Chilpa et al. 2004; Yasunaka et al. 2005). Genus Calophyllum is a rich source of various triterpenes, which belong to various groups like adianane [ $3 \beta$-simiarenol (114)], fridelane [frideline (115) and canophyllol (116)], lupane [betulinic acid (111)], oleanane [canophyllicacid (117) and apetalactone (118)] and taraxerane [taraxerol (119) and taraxerone (120)]. Furthermore, stigmasterol (121) was also isolated from the stem bark of C. wallichianum (Tee et al. 2018) (Fig. 5.1; Table 5.2).

\subsubsection{Glycosides}

Flavonoid glycoside, myricetin-7-glucoside (128), was isolated from the androecium of flowers of $C$. inophyllum (Subramanian and Nair 1971). Similarly, Ming et al. (2016) isolated a new C-glycoside calophymembranside C (124) from the stem of C. membranaceum. Further, Zhu et al. (2018) isolated three C-glycosides calophymembranside $\mathrm{D}(\mathbf{1 2 5})$, calophymembranside $\mathrm{E} \quad$ (126) and calophymembranside F (127), from the stem of C. membranaceum (Fig. 5.1).

\subsubsection{Miscellaneous}

Secondary metabolites belonging to other chemical classes apart from the abovementioned ones have also been isolated from the genus Calophyllum. $\mathrm{A} \mathrm{C}_{24}$ terpenoid, soulattrone A, was isolated from the bark of $C$. soulattri (Nigam et al. 1988). Enervosanone (129) and cambogin (130), two phloroglucinols, were isolated from the stem bark of C. enervosum (Taher et al. 2005). Similarly, two 3-propylpropanoic acid moiety-bearing phloroglucinols, i.e. sundaicumone A (131) and sundaicumone B (132), were isolated in bioassay-guided fractionation using glucocorticoid receptor assay from the leaves of $C$. sundaicum (Cao et al. 2006a, b) (Fig. 5.1; Table 5.2).

\subsection{Bioactivities of Genus Calophyllum}

The genus Calophyllum exhibited several biological activities such as antiviral, antimalarial, chemopreventive, antisecretory, antibacterial, cytoprotective, analgesic, antitumour-promoting and cytotoxic activity.

\subsubsection{Antiviral Activity}

The dipyrano-tetracyclic coumarins such as calanolides, inophyllums and cordatolides isolated from the genus have exhibited potential anti-HIV activity (Kashman et al. 1992; Patil et al. 1993; Dharmaratne et al. 1998a, b). Studies on its mechanism of action showed that these compounds inhibit reverse transcriptase enzyme and are classified as non-nucleoside reverse transcriptase inhibitors (Creagh 
et al. 2001). Researchers from the National Cancer Institute studied anticancer potential of Malaysian trees in Sarawak's forest. Unfortunately, they did not get any anticancer compound; instead they found an anti-HIV compound calanolide A from leaves and twigs of $C$. lanigerum. The isolated compound calanolide A showed complete protection against HIV-1 replication, with an $\mathrm{IC}_{50}$ of $5.9 \pm 1.9 \mu \mathrm{M}$. The relocation efforts of the tree were failed and percentage of calanolide A was very less in other species of the genus Calophyllum. Further research showed that calanolide B, an isomer of calanolide A, is slightly less active. Calanolide B has the advantage of being readily available from the latex without causing any harm to the trees (Kashman et al. 1992). The development of calanolides was licensed by $\mathrm{NCI} / \mathrm{NIH}$ to MediChem Research, Inc. (now Advanced Life Sciences) which negotiated an agreement with the Sarawak State Government for the development of calanolides as an anti-HIV drug. A joint venture company named Sarawak Medichem Pharmaceuticals was incorporated for the development of the lead molecule. After the joint venture arrangement proved unworkable, the lead role in the development was transferred to a Sarawakian company named Craun Research Sendirian Berhad. MediChem Research successfully synthesized (+)-calanolide A which is in early clinical trials, while $(-)$-calanolide B is in preclinical development. 11-Demethyl-12-oxo, an analogue of calanolide A, possesses comparable in vitro anti-HIV-1 activity and is used as a template to study structure-activity relationship of other congeners (Hanna 1999).

Inophyllums such as inophyllum B and $\mathrm{P}$, isolated from $C$. inophyllum, displayed activity against $\mathrm{HIV}$ with an $\mathrm{IC}_{50}$ value of $38 \mathrm{nM}$ and $130 \mathrm{nM}$, respectively (Patil et al. 1993). Similarly, cordatolides A and B isolated from C. cordato-oblongum showed anti-HIV activity with an $\mathrm{IC}_{50}$ value of $12.3 \mathrm{nM}$ and $19 \mu \mathrm{M}$, respectively. Five pyranoxanthones isolated from $C$. blancoi also showed activity against the coronavirus with $\mathrm{EC}_{50}$ of 3-15 $\mu \mathrm{g} / \mathrm{ml}$ (Shen et al. 2005) (Table 5.3).

\subsubsection{Antimicrobial Activity}

Undi oil (C. inophyllum) showed antibacterial activity against several Gram-positive bacteria. The activity of ethanol extract was 14 times of the original oil (Bhat et al. 1954). Novel chromanone acids, brasiliensophyllic acid A-C and isobrasiliensophyllic acid A-C, isolated from the bark of $C$. brasiliense exhibited antibacterial activity against Bacillus cereus and Staphylococcus epidermidis (Cottiglia et al. 2004). In addition, different parts of $C$. soulattri plant (methyl alcohol extracts of root, stem bark and leaf barks) exhibited a wide range of antibacterial activities (Khan et al. 2002) (Table 5.3).

\subsubsection{Inhibition of the Multidrug Transporter P-glycoprotein}

The coumarins of genus Calophyllum as well as their synthetic analogues inhibited the multidrug transporter P-glycoprotein. Structure-activity relationship study of 
Table 5.3 Bioactive compounds isolated from Calophyllum spp.

\begin{tabular}{|c|c|c|}
\hline Compound & Property/active against & Reference(s) \\
\hline \multicolumn{3}{|l|}{ Antiviral compounds } \\
\hline Calanolide F & Anti-HIV & McKee et al. (1996) \\
\hline (-)-Calanolide B & Anti-HIV & McKee et al. (1996) \\
\hline Calanolide A & Anti-HIV & Kashman et al. (1992) \\
\hline Calophyllolide & Anticoagulant & Arora et al. (1962) \\
\hline Inophyllum B, P & HIV-RT inhibition & Patil et al. (1993) \\
\hline Soulattrolide & HIV-RT inhibition & Pengsuparp et al. (1996) \\
\hline Cordatolide A, B & HIV-RT inhibition & $\begin{array}{l}\text { Dharmaratne et al. } \\
(1998 \mathrm{a}, \mathrm{b})\end{array}$ \\
\hline Inophyllum B, P, D, C & HIV-RT inhibition & Patil et al. (1993) \\
\hline Inophyllum B acetate & HIV-RT inhibition & Patil et al. (1993) \\
\hline 11,12-Anhydroinophyllum P & HIV-RT inhibition & Patil et al. (1993) \\
\hline \multicolumn{3}{|l|}{ Antimicrobial compounds } \\
\hline Dicaledol & A. fumigatus & $\begin{array}{l}\text { Morel et al. (2002); Oger } \\
\text { et al. (2003) }\end{array}$ \\
\hline Caledonixanthone E & A. fumigatus & Morel et al. (2002) \\
\hline Caloxanthone $\mathrm{F}$ & A. fumigatus & Morel et al. (2002) \\
\hline $\begin{array}{l}\text { 7-Hydroxy-1,8- } \\
\text { dimethoxyxanthone }\end{array}$ & A. fumigatus & Morel et al. (2002) \\
\hline Calolongic acid & A. fumigatus & Hay et al. (2003) \\
\hline Isocalolongic acid & A. fumigatus & Hay et al. (2003) \\
\hline Calanolide E & B. cereus & Tee et al. (2018) \\
\hline Brasiliensophyllic acid A, B & B. cereus & Cottiglia et al. (2004) \\
\hline Isobrasiliensophyllic acid A, B & B. cereus & Cottiglia et al. (2004) \\
\hline Calanolide E & $\begin{array}{l}\text { B. subtilis, B. megaterium, } \\
\text { B. pumilus }\end{array}$ & Tee et al. (2018) \\
\hline Blancoxanthone & Coronavirus & Shen et al. (2005) \\
\hline Cambogin & $\begin{array}{l}\text { B. subtilis, E. coli, } \\
\text { P. aeruginosa, S. aureus }\end{array}$ & Taher et al. (2005) \\
\hline Enervosanone & $\begin{array}{l}\text { B. subtilis, E. coli, S. aureus, } \\
\text { P. aeruginosa }\end{array}$ & Taher et al. (2005) \\
\hline Calozeyloxanthone & E. faecalis, E. faecium (VR) & Sakagami et al. (2002) \\
\hline Mammea A/BB & M. tuberculosis H37Rv & Pires et al. (2014) \\
\hline$(-)$ Mammea B/BB & M. tuberculosis H37Rv & Pires et al. (2014) \\
\hline Mammea A/BA & S. aureus & Yasunaka et al. (2005) \\
\hline Jacareubin & S. aureus & Yasunaka et al. (2005) \\
\hline $\begin{array}{l}\text { 1,3,5,6-Tetrahydroxy-2- } \\
\text { (3,3-dimethylallyl)xanthone }\end{array}$ & S. aureus & Yasunaka et al. (2005) \\
\hline Calophyllolide & S. aureus & $\begin{array}{l}\text { Bhat et al. (1954); Yimdjo } \\
\text { et al. (2004) }\end{array}$ \\
\hline Caloxanthone A & S. aureus & Yimdjo et al. (2004) \\
\hline Calophynic acid & S. aureus & Yimdjo et al. (2004) \\
\hline Brasiliensic acid & S. aureus & Yimdjo et al. (2004) \\
\hline Inophylloidic acid & S. aureus & Yimdjo et al. (2004) \\
\hline
\end{tabular}


Table 5.3 (continued)

\begin{tabular}{l|l|l}
\hline Compound & Property/active against & Reference(s) \\
\hline Calaustralin & S. aureus & Yimdjo et al. (2004) \\
\hline Inophyllum C & S. aureus & Yimdjo et al. (2004) \\
\hline Calozeloxanthone & S. aureus (MR) & Dharmaratne et al. (1999) \\
\hline Calozeloxanthone & S. aureus (MR) & Yasunaka et al. (2005) \\
\hline A
\end{tabular}

\section{Anticancer compounds}

TPA-induced EBV-EA activation inhibitory activity

\begin{tabular}{|c|c|c|}
\hline Brasixanthone B & - & Ito et al. (2002) \\
\hline Brasixanthone $\mathrm{C}$ & - & Ito et al. (2002) \\
\hline Brasixanthone D & - & Ito et al. (2002) \\
\hline 8-Desoxygartanin & - & Ito et al. (2002) \\
\hline Calanolide A & - & Ito et al. (2003) \\
\hline Brasimarin A & - & Ito et al. (2003) \\
\hline Brasimarin B & - & Ito et al. (2003) \\
\hline Brasimarin $\mathrm{C}$ & - & Ito et al. (2003) \\
\hline Calanolide $\mathrm{C}$ & - & Ito et al. (2003) \\
\hline Calanone & - & Ito et al. (2003) \\
\hline Garcinianin & - & Ito et al. (1999) \\
\hline Mammea B/BB & - & Ito et al. (2003) \\
\hline Talbotaflavone & - & Ito et al. (1999) \\
\hline Calocoumarin A & - & Itoigawa et al. (2001) \\
\hline Isocalophyllic acid & - & Itoigawa et al. (2001) \\
\hline Calophyllolide & - & Itoigawa et al. (2001) \\
\hline Apetatolide & - & Itoigawa et al. (2001) \\
\hline \multicolumn{3}{|l|}{ Cytotoxic compounds } \\
\hline $\begin{array}{l}\text { Hexane extract of } C . \text { mucigerum } \\
\text { stem bark }\end{array}$ & CEM-SS & Ee et al. (2004) \\
\hline Apetalic acid methyl ester & Hela, KB, Med & Shen et al. (2004) \\
\hline Apetalic acid 5-O-acetate & Hela, KB, Med & Shen et al. (2004) \\
\hline Isopetalic acid methyl ester & Hela, KB, Med & Shen et al. (2004) \\
\hline Isorecedensolide & Hela, KB, Med & Shen et al. (2004) \\
\hline Recedensolide & Hela, KB, Med & Shen et al. (2004) \\
\hline Mammea A/BA & $\mathrm{k} 562, \mathrm{pc} 3, \mathrm{u} 251$ & Reyes-Chilpa et al. (2004) \\
\hline Mammea $\mathrm{C} / \mathrm{OA}+\mathrm{C} / \mathrm{OB}$ & $\mathrm{k} 562, \mathrm{pc} 3, \mathrm{u} 251$ & Reyes-Chilpa et al. (2004) \\
\hline Mammea A/AA cyclo F & KB & Guilet et al. (2001) \\
\hline Mammea A/AB cyclo F & KB & Guilet et al. (2001) \\
\hline Mammea A/AC cyclo $F$ & KB & Guilet et al. (2001) \\
\hline Caloxanthone A & KB & Ito et al. (2002) \\
\hline Calophynic acid & KB & Ito et al. (2002) \\
\hline Brasiliensic acid & $\mathrm{KB}$ & Ito et al. (2002) \\
\hline Inophylloidic acid & KB & Ito et al. (2002) \\
\hline Calophyllolide & KB & Ito et al. (2002) \\
\hline Pyranojacareubin & KB & Ito et al. (2002) \\
\hline
\end{tabular}

$V R$ vancomycin resistant, $M R$ methicillin resistant 
these compounds showed a favourable region of electrostatic and steric volume. The study also revealed the importance of hydrophobic and neutral-charge group for the activity (Raad et al. 2006).

\subsubsection{Anticancer Activity}

Mammea-type coumarins (A/BA, A/BB, B/BB and B/BA) showed anticancer activity against various cancer cell lines including BV173, HL60, HTC116, K562, MALM6, PC3, SEM, U251 and a P-glycoprotein overexpressing cell line. Study of their mechanism of action suggested that these anticancer activities are due to the induction of caspase-mediated cell death (Kimura et al. 2005). Brasixanthones A-D displayed significant anti-proliferative activity against TPA-induced Epstein-Barr virus early antigen (EBV-EA) activation in Raji cells lines (Ito et al. 2002). Similarly, 8-desoxygartanin, calanolide A, brasimarin A, brasimarin B, brasimarin $\mathrm{C}$, calanolide $\mathrm{C}$, calanone and mammea $\mathrm{B} / \mathrm{BB}$ also showed inhibition of TPA-induced EBV-EA activation in Raji cell lines (Ito et al. 2003) (Table 5.3).

\subsubsection{Antimalarial Activity}

Hay et al. (2004) isolated and tested seven xanthone compounds against chloroquino-resistant strain of Plasmodium falciparum. The $\mathrm{IC}_{50}$ values of tested compounds range from 0.8 to $4.4 \mu \mathrm{g} / \mathrm{ml}$. SAR study showed that the $\mathrm{OH}$ group position is critical for the activity and the presence of 1,1-dimethylallyl, an additional pyran ring, two isopentenyl chains or one isopentenyl chain with a pyranic ring is favourable for the activity. It was also concluded that the hydroxylation of the prenyl side chain is not required for higher activity. The resin of $C$. antillanum showed potent activity against $P$. falciparum with an $\mathrm{IC}_{50}$ value of $0.3 \mu \mathrm{g} / \mathrm{ml}$ (Cuesta-Rubio et al. 2015).

\subsubsection{Anti-parasite Activity}

Mammea-type coumarins (A/BA, A/BB and B/BB) showed anti-parasitic activity against Trypanosoma cruzi and Leishmania amazonensis. The observed antiparasitic activity was due to disruption of mitochondrial swelling, which in turn loses normal ultrastructure (Reyes-Chilpa et al. 2004). Similarly, three xanthones (jacareubin, 6-deoxyjacareubin, and 1,3,5,6-tetrahydroxy-2-(3-methyl-2-butenyl) xanthone) isolated from the heartwood of $C$. brasiliense showed in vitro trypanocidal activity against epimastigotes and trypomastigotes of $T$. cruzi. Further, xanthones isolated from $C$. brasiliense showed potential against Chagas disease with $\mathrm{IC}_{100}$ value of $153-213 \mu \mathrm{M}$ against trypomastigotes (Abe et al. 2004). 


\subsubsection{Sulphotransferase Inhibitor}

Xanthones isolated from the heartwood of $C$. brasiliense showed reversible inhibition of sulphotransferases $1 \mathrm{~A} 1$ (SULT1A1) with $\mathrm{IC}_{50}$ value ranging from 1.6 to $7.4 \mu \mathrm{M}$. Similarly, coumarins isolated from $C$. brasiliense showed inhibition of SULT1A1 with $\mathrm{IC}_{50}$ value ranging from 47 to $185 \mu \mathrm{M}$ and SULT2A1 with $\mathrm{IC}_{50}$ value ranging from 16 to $31 \mu \mathrm{M}$ (Mesía-Vela et al. 2001).

\subsubsection{Anti-dyslipidaemic Activity}

The canophyllic acid, amentoflavone and a mixture of calophyllic acid and isocalophyllic acid isolated from $C$. inophyllum showed dose-dependent lipid-lowering activity under in vivo condition in triton-induced hyperlipidaemia model (Prasad et al. 2012).

\subsubsection{Antioxidant Activity and Anti-inflammatory Activity}

Oil obtained from the nuts of $C$. inophyllum showed antioxidant activity by inhibiting lipid peroxidation. The antioxidant activity of the oil helps to protect skin cells from damage by reactive oxygen species (Mahmud et al. 1998). Xanthones isolated form $C$. inophyllum exhibited in vivo anti-inflammatory activity when administered through intraperitoneal or oral routes in rats (Gopalakrishnan et al. 1980).

\subsubsection{Hypotensive Activity}

Oku et al. (2005) studied inhibitory effects of 22 xanthones on exogenous plateletactivating factor (PAF)-induced hypotension in in vivo assay. The result of the study showed that caloxanthone E, 1,3,5,6-tetrahydroxy-2-isoprenylxanthone, 6-deoxyjacareubin and guanidine showed 60\% inhibition in PAF-induced hypotension (Oku et al. 2005).

\subsubsection{1 $\alpha$-Glucosidase Activity}

Two flavonoids amentoflavone and carpachromene along with two coumarins, inophyllum $\mathrm{D}$ and inophyllum $\mathrm{H}$, isolated from the crude extracts of the bark and leaves of $C$. symingtonianum showed promising $\alpha$-glucosidase activity with $\mathrm{IC}_{50}$ ranging from 6.4 to $62.3 \mu \mathrm{M}$, which was better than the synthetic drug acarbose $\left(\mathrm{IC}_{50}\right.$ $456.4 \mu \mathrm{M})$ (Aminudin et al. 2015). 


\subsubsection{Other Activities}

Coumarin named inophyllolide isolated by bioassay-guided fractionation from the nuts of $C$. inophyllum showed anti-piscicidal activity (Kawazu et al. 1968). Calofloride isolated from the seeds of $C$. verticillatum showed significant molluscicidal activity (Ravelonjato et al. 1992). Calophyllolide, a coumarin isolated from the $C$. inophyllum, showed anticoagulant action in in vivo experiments. The coagulation activity was in between the dicoumarol (slow and long acting) and ethyl biscoumacetate (very fast and short acting) (Arora et al. 1962). Amentoflavone isolated from the bark and leaves of $C$. symingtonianum showed potential 15-LOX inhibitory activity with an $\mathrm{IC}_{50}$ value of $0.04 \mu \mathrm{M}$ (Aminudin et al. 2015).

\subsection{Conclusions}

Genus Calophyllum is a rich source of bioactive secondary metabolites of class xanthones, coumarins and chromanone. Other classes of secondary metabolites like triterpenoid and glycoside found in the genus Calophyllum have shown a wide range of biological activities like antiviral, anticancer, antimalarial, antibacterial and antiproliferative and inhibition of P-glycoprotein (involved in the multidrug transport process) and inhibition of sulphotransferases. Calanolide A, isolated from the genus Calophyllum, has shown potential anti-HIV activity and continues to be in the clinical developmental stage. The dependence of calanolide A availability on isolation from the natural source has posed a problem in its further development. Various synthetic routes and alternative sources of the compounds with better yields are being explored to overcome this problem. Further studies to explore the molecular mechanism of the phytochemicals need to be done to exploit it for health benefits.

Conflict of Interest Authors declare no conflict of interest.

\section{References}

Abe F, Nagafuji S, Okabe H, Akahane H, Estrada-Muñiz E, Huerta-Reyes M, Reyes-Chilpa R (2004) Trypanocidal constituents in plants 3. Leaves of Garcinia intermedia and heartwood of Calophyllum brasiliense. Biol Pharm Bull 27(1):141-143

Abraham E. Materia Medica Guian. Britt. Timehri, 3rd ser. 1912;2

Ali MS, Mahmud S, Perveen S, Ahmad VU, Rizwani GH (1999) Epimers from the leaves of Calophyllum inophyllum. Phytochemistry 50(8):1385-1389

Aminudin NI, Ahmad F, Taher M, Zulkifli RM (2015) $\alpha$-Glucosidase and 15-lipoxygenase inhibitory activities of phytochemicals from Calophyllum symingtonianum. Nat Prod Commun 10 (9): $1585-1587$

Arora R, Mathur C, Seth S (1962) Calophyllolide, a complex coumarin anticoagulant from Calophyllum inophyllum Linn. J Pharm Pharmacol 14(1):534-535

Babu V, Arya R, Ilyas M, Nasim K (1994) 9-Hydroxy-2, 2, 6, 7-tetramethyl-2H-[1]-benzopyran(1-phenylethylene-10-yl)-[3, 2-b]-dihydropyran-4-one from Calophyllum tomentosum. Phytochemistry 35(2):507-510 
Bhat SG, Kane JG, Sreenivasan A (1954) The in vitro evaluation of the antibacterial activity of Undi oil (Calophyllum inophyllum Linn.). J Am Pharm Assoc 43(9):543-546

Cao S, Low K-N, Glover RP, Crasta SC, Ng S, Buss AD, Butler MS (2006a) Sundaicumones a and B, Polyprenylated Acylphloroglucinol derivatives from Calophyllum sundaicum with weak activity against the glucocorticoid receptor. J Nat Prod 69(4):707-709

Cao S-G, Sim KY, Goh S (2001) Minor methylated pyranoamentoflavones from Calophyllum venulosum. Nat Prod Lett 15(5):291-297

Cao S-G, Sim KY, Goh SH (2006b) Minor methylated Pyranoamentoflavones from Calophyllum venulosum. Nat Prod Lett 15(5):291-297

Cottiglia F, Dhanapal B, Sticher O, Heilmann J (2004) New chromanone acids with antibacterial activity from Calophyllum brasiliense. J Nat Prod 67(4):537-541

Creagh T, Ruckle JL, Tolbert DT, Giltner J, Eiznhamer DA, Dutta B, Flavin MT, Xu Z-Q (2001) Safety and pharmacokinetics of single doses of (+)-Calanolide a, a novel, naturally occurring nonnucleoside reverse transcriptase inhibitor, in healthy, human immunodeficiency virusnegative human subjects. Antimicrob Agents Chemother 45(5):1379-1386

Cuesta-Rubio O, Oubada A, Bello A, Maes L, Cos P, Monzote L (2015) Antimicrobial assessment of resins from Calophyllum antillanum and Calophyllum inophyllum. Phytother Res 29 (12):1991-1994

Dahanayake M, Kitagawa I, Somanathan R, Sultanbawa MUS (1974) Chemical investigation of ceylonese plants. Part VII. Extractives of Calophyllum thwaitesii planch and Triana and Calophyllum walkeri Wight (Guttiferae). J Chem Soc, Perkin Trans 1:2510-2514

Dharmaratne HRW, Sajeevani JRDM, Marasinghe GPK, Ekanayake EMHGS (1998a) Distribution of pyranocoumarins in Calophyllum cordato-oblongum. Phytochemistry 49(4):995-998

Dharmaratne HRW, Wanigasekera WMAP, Mata-Greenwood E, Pezzuto JM (1998b) Inhibition of human immunodeficiency virus type 1 reverse transcriptase activity by Cordatolides isolated from Calophyllum cordato-oblongum. Planta Med 64(05):460-461

Dharmaratne HRW, Wijesinghe WMNM, Thevanasem V (1999) Antimicrobial activity of xanthones from Calophyllum species, against methicillin-resistant Staphylococcus aureus (MRSA). J Ethnopharmacol 66(3):339-342

Dharmaratne HRW, Wijesinghe WNM (1997) A trioxygenated diprenylated chromenxanthone from Calophyllum moonii. Phytochemistry 46(7):1293-1295

Díaz DMV (2013) Multivariate analysis of morphological and anatomical characters of Calophyllum (Calophyllaceae) in South America. Bot J Linn Soc 171(3):587-626

Dorla E, Grondin I, Hue T, Clerc P, Dumas S, Gauvin-Bialecki A, Laurent P (2019) Traditional uses, antimicrobial and acaricidal activities of 20 plants selected among Reunion Island's flora. $\mathrm{S}$ Afr J Bot 122:447-456

Eckenwalder JE (1980) Taxonomy of the West Indian cycads. J Arnold Arbor 61(4):701-722

Ee GCL, Ng KN, Taufiq-Yap YH, Rahmani M, Ali AM, Muse R (2004) Mucigerin, a new coumarin from Calophyllum mucigerum (Guttiferae). Nat Prod Res 18(2):123-128

Gomez-Verjan JC, Estrella-Parra EA, González-Sánchez I, Vázquez-Martínez ER, VergaraCastañeda E, Cerbón MA, Reyes-Chilpa R (2014) Molecular mechanisms involved in the cytotoxicity induced by coumarins from $\mathrm{C}$ alophyllum brasiliense in $\mathrm{K} 562$ leukaemia cells. J Pharm Pharmacol 66(8):1189-1195

Gopalakrishnan C, Shankaranarayanan D, Nazimudeen S, Viswanathan S, Kameswaran L (1980) Anti-inflammatory and CNS depressant activities of xanthones from Calophyllum inophyllum and Mesua ferrea. Indian J Pharmacol 12(3):181

Govindachari T, Prakash D, Viswanathan N (1968) Apetalactone, a new triterpene lactone from Calophyllum species. J Chem Soc C: Organic:1323-1324

Govindachari TR, Viswanathan N, Pai BR, Rao UR, Srinivasan M (1967) Triterpenes of Calophyllum inophyllum Linn. Tetrahedron 23(4):1901-1910

Grenand P, Moretti C, Jacquemin H. Pharmacopées Traditionnelles en Guyane, Créoles, Palikur. Wayapi. Edit. ORSTOM, Paris. 1987 
Group AP (2009) An update of the angiosperm phylogeny group classification for the orders and families of flowering plants: APG III. Bot J Linn Soc 161(2):105-121

Guerreiro E, Kunesch G, Polonsky J (1973) Chromanones de l'écorce de Calophyllum recedens. Phytochemistry 12(1):185-189

Guilet D, Seraphin D, Rondeau D, Richomme P, Bruneton J (Oct 2001) Cytotoxic coumarins from Calophyllum dispar. Phytochemistry 58(4):571-575

Gunasekera SP, Jayatilake GS, Selliah SS, Sultanbawa MUS (1977) Chemical investigation of ceylonese plants. Part 27. Extractives of Calophyllum cuneifolium Thw. And Calophyllum soulattri Burm. F.(Guttiferae). J Chem Soc, Perkin Trans 1 13:1505-1511

Hanna L (1999) Calanolide A: a natural non-nucleoside reverse transcriptase inhibitor. BETA 12 (2):8

Hay AE, Guilet D, Morel C, Larcher G, Macherel D, Le Ray AM, Litaudon M, Richomme P (2003) Antifungal Chromans inhibiting the mitochondrial respiratory chain of pea seeds and new Xanthones from Calophyllum caledonicum. Planta Med 69(12):1130-1135

Hay A-E, Hélesbeux J-J, Duval O, Labaïed M, Grellier P, Richomme P (2004) Antimalarial xanthones from Calophyllum caledonicum and Garcinia vieillardii. Life Sci 75(25):3077-3085

Iinuma M, Ito T, Tosa H, Tanaka T, Miyake R, Chelladurai V (1997) Prenylated xanthonoids from Calophyllum apetalum. Phytochemistry 46(8):1423-1429

Iinuma M, Tosa H, Tanaka T, Yonemori S (1995) Two xanthones from roots of Calophyllum inophyllum. Phytochemistry 38(3):725-728

Iinuma M, Tosa H, Toriyama N, Tanaka T, Ito T, Chelladurai V (1996) Six xanthones from Calophyllum austroindicum. Phytochemistry 43(3):681-685

Ito C, Itoigawa M, Mishina Y, Filho VC, Enjo F, Tokuda H, Nishino H, Furukawa H (2003) Chemical constituents of Calophyllum brasiliense. 2. Structure of three new coumarins and cancer chemopreventive activity of 4-substituted coumarins. J Nat Prod 66(3):368-371

Ito C, Itoigawa M, Mishina Y, Filho VC, Mukainaka T, Tokuda H, Nishino H, Furukawa H (2002) Chemical constituents of Calophyllum brasiliensis: structure elucidation of seven new Xanthones and their cancer Chemopreventive activity. J Nat Prod 65(3):267-272

Ito C, Itoigawa M, Miyamoto Y, Rao KS, Takayasu J, Okuda Y, Mukainaka T et al (1999) A new Biflavonoid from Calophyllum panciflorum with antitumor-promoting activity. J Nat Prod 62 (12):1668-1671

Itoigawa M, Ito C, Tan HT-W, Kuchide M, Tokuda H, Nishino H, Furukawa H (2001) Cancer chemopreventive agents, 4-phenylcoumarins from Calophyllum inophyllum. Cancer Lett 169 (1): $15-19$

Joshi SP, Kulkarni SR, Phalgune UD, Puranik VG (2013) New dipyranocoumarin from the leaves of Calophyllum apetalum Willd. Nat Prod Res 27(20):1896-1901

Karunanayake S, Sotheeswaran S, Uvais M, Sultanbawa S, Balasubramaniam S (1981) Xanthones and triterpenes of Calophyllum tomentosum. Phytochemistry 20(6):1303-1304

Kashman Y, Gustafson KR, Fuller RW, Cardellina JH, McMahon JB, Currens MJ, Buckheit RW et al (1992) The calanolides, a novel HIV-inhibitory class of coumarin derivatives from the tropical rainforest tree, Calophyllum lanigerum. J Med Chem 35(15):2735-2743

Kawazu K, Ohigashi H, Mitsui T (1968) The piscicidal constituents of Calophyllum inophyllum Linn. Tetrahedron Lett 9(19):2383-2385

Khan M, Kihara M, Omoloso A (2002) Antimicrobial activity of Calophyllum soulattri. Fitoterapia 73(7-8):741-743

Khan NU-D, Parveen N, Singh MP, Singh R, Achari B, Dastidar PP, Dutta PK (1996) Two isomeric benzodipyranone derivatives from Calophyllum inophyllum. Phytochemistry 42(4):1181-1183

Kimura S, Ito C, Jyoko N, Segawa H, Kuroda J, Okada M, Adachi S et al (2005) Inhibition of leukemic cell growth by a novel anti-cancer drug (GUT-70) from Calophyllum brasiliense that acts by induction of apoptosis. Int J Cancer 113(1):158-165

Lavergne R. Tisaneurs et plantes médicinales indigènes: l'île de La Réunion: Éd. Orphie; 2001

Mah SH, Cheng Lian Ee G, Teh SS, Sukari MA (2015) Antiproliferative xanthone derivatives from Calophyllum inophyllum and Calophyllum soulattri. Pak J Pharm Sci 28(2):425-429 
Mahmud S, Rizwani G, Ahmad M, Ali S, Perveen S, Ahmad V (1998) Antimicrobial studies on fractions and pure compounds of Calophyllum inophyllum Linn. Pak J Pharmacol 15(2):13-25

McKee TC, Fuller RW, Covington CD, Cardellina JH, Gulakowski RJ, Krepps BL, McMahon JB, Boyd MR (1996) New pyranocoumarins isolated from Calophyllum lanigerum and Calophyllum teysmannii. J Nat Prod 59(8):754-758

Mesía-Vela S, Sańchez R, Estrada-Muniz E, Alavez-Solano D, Torres-Sosa C, Jiménez-Estrada M, Reyes-Chilpa R, Kauffman F (2001) Natural products isolated from Mexican medicinal plants: novel inhibitors of sulfotransferases, SULT1A1 and SULT2A1. Phytomedicine 8(6):481-488

Ming M, Zhang X, Chen H-F, Zhu L-J, Zeng D-Q, Yang J, Wu G-X, Wu Y-Z, Yao X-S (2016) $\mathrm{RXR} \alpha$ transcriptional inhibitors from the stems of Calophyllum membranaceum. Fitoterapia 108:66-72

Morel C, Séraphin D, Teyrouz A, Larcher G, Bouchara J-P, Litaudon M, Richomme P, Bruneton J (2002) New and antifungal xanthones from Calophyllum caledonicum. Planta Med 68 (01):41-44

Nigam S, Mitra C (1968) Constituents of Calophyllum tomentosum and C. apetalum nuts. Planta Med 16(04):450-457

Nigam S, Mitra C (1969) Constituents of Calophyllum apetalum and Calophyllum tomentosum trunk bark. Phytochemistry 8(01):223-224

Nigam SK, Banerji R, Rebuffat S, Pascard C, Bodo B (1988) Soulattrone a, a C24 terpenoid from Calophyllum soulattri. Phytochemistry 27(2):527-530

Oger J-M, Morel C, Helesbeux J-J, Litaudon M, Séraphin D, Dartiguelongue C, Larcher G, Richomme P, Duval O (2003) First 2-hydroxy-3-methylbut-3-enyl substituted xanthones isolated from plants: structure elucidation, synthesis and antifungal activity. Nat Prod Res 17 (3):195-199

Oku H, Ueda Y, Iinuma M, Ishiguro K (2005) Inhibitory effects of xanthones from Guttiferae plants on PAF-induced hypotension in mice. Planta Med 71(01):90-92

Ormancey-Potier A, Buzas A, Lederer E (1951) Sur le calophyllolide et $1^{\prime}$ acide calophyllique isolés des graines de Calophyllum inophyllum. Bulletin de la Société Chimique de France. 577

Patil AD, Freyer AJ, Eggleston DS, Haltiwanger RC, Bean MF, Taylor PB, Caranfa MJ, Breen AL, Bartus HR (1993) The inophyllums, novel inhibitors of HIV-1 reverse transcriptase isolated from the Malaysian tree, Calophyllum inophyllum Linn. J Med Chem 36(26):4131-4138

Pengsuparp T, Serit M, Hughes SH, Soejarto DD, Pezzuto JM (1996) Specific inhibition of human immunodeficiency virus type 1 reverse transcriptase mediated by soulattrolide, a coumarin isolated from the latex of Calophyllum teysmannii. J Nat Prod 59(9):839-842

Pires CTA, Brenzan MA, Scodro RB, DAG C, LDG L, VLD S, Cardoso RF (2014) Antimycobacterium tuberculosis activity and cytotoxicity of Calophyllum brasiliense Cambess (Clusiaceae). Mem Inst Oswaldo Cruz 109(3):324-329

Polonsky J (1957) Structure Chimique Du Calophyllolide, De Linophyllolide Et De Lacide Calophyllique, Constituants Des Noix De Calophyllum inophyllum. Bull Soc Chim Fr 7:1079-1087

Prasad J, Shrivastava A, Khanna A, Bhatia G, Awasthi S, Narender T (2012) Antidyslipidemic and antioxidant activity of the constituents isolated from the leaves of Calophyllum inophyllum. Phytomedicine 19(14):1245-1249

Raad I, Terreux R, Richomme P, Matera E-L, Dumontet C, Raynaud J, Guilet D (2006) Structureactivity relationship of natural and synthetic coumarins inhibiting the multidrug transporter P-glycoprotein. Bioorg Med Chem 14(20):6979-6987

Ravelonjato B, Libot F, Ramiandrasoa F, Kunesch N, Gayral P, Poisson J (1992) Molluscicidal constituents of Calophyllum from Madagascar: activity of some natural and synthetic neoflavonoids and khellactones. Planta Med 58(01):51-55

Reyes-Chilpa R, Estrada-Muñiz E, Apan TR, Amekraz B, Aumelas A, Jankowski CK, VázquezTorres M (2004) Cytotoxic effects of mammea type coumarins from Calophyllum brasiliense. Life Sci 75(13):1635-1647 
Sakagami Y, Kajimura K, Wijesinghe W, Dharmaratne H (2002) Antibacterial activity of calozeyloxanthone isolated from Calophyllum species against vancomycin-resistant enterococci (VRE) and synergism with antibiotics. Planta Med 68(06):541-543

Shen Y-C, Hung M-C, Wang L-T, Chen C-Y (2003) Inocalophyllins A, B and their methyl esters from the seeds of Calophyllum inophyllum. Chem Pharm Bull 51(7):802-806

Shen Y-C, Wang L-T, Khalil AT, Chiang LC, Cheng P-W (2005) Bioactive pyranoxanthones from the roots of Calophyllum blancoi. Chem Pharm Bull 53(2):244-247

Shen Y-C, Wang L-T, Khalil AT, Kuo Y-H (2004) Chromanones and dihydrocoumarins from Calophyllum blancoi. Chem Pharm Bull 52(4):402-405

Stevens PF (1980) A revision of the Old World species of Calophyllum (Guttiferae). J Arnold Arbor 61(2):117-424

Subramanian SS, Nair A (1971) Myricetin-7-Glucoside from the androecium of the flowers of Calophyllum inophyllum. Phytochemistry 10(7):1679-1680

Taher M, Idris MS, Ahmad F, Arbain D (2005) A polyisoprenylated ketone from Calophyllum enervosum. Phytochemistry 66(6):723-726

Tee KH, Ee GCL, Ismail IS, Karunakaran T, Teh SS, Jong VYM, Mohd Nor SM (2018) A new coumarin from stem bark of Calophyllum wallichianum. Nat Prod Res 32(21):2565-2570

Watt G (2014) Dictionary of the economic products of India volume II (Cabbage to Cyperus). Cambridge University Press, Cambridge, pp 29-32

Yasunaka K, Abe F, Nagayama A, Okabe H, Lozada-Pérez L, López-Villafranco E, Muñiz EE, Aguilar A, Reyes-Chilpa R (2005) Antibacterial activity of crude extracts from Mexican medicinal plants and purified coumarins and xanthones. J Ethnopharmacol 97(2):293-299

Yimdjo MC, Azebaze AG, Nkengfack AE, Meyer AM, Bodo B, Fomum ZT (2004) Antimicrobial and cytotoxic agents from Calophyllum inophyllum. Phytochemistry 65(20):2789-2795

Zhu L-J, Yi S, Li X, Chen H-F, Ming M, Zhang X, Yao X-S (2018) C-glycosides from the stems of Calophyllum membranaceum. J Asian Nat Prod Res 20(1):49-54 